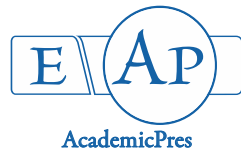

\title{
An Ethnobotanical Study of Plant Species Used for Medicine by the Eegun Indigenous Tribal Group of Lagos State, Nigeria
}

\author{
Damilola Eunice ADEDEJI*, Joshua KAYODE, Modupe Janet AYENI
}

\author{
Ekiti State University, Department of Plant Science and Biotechnology, Ado-Ekiti,
}

Nigeria; adedejieunice91@yahoo.com (* corresponding author)

\begin{abstract}
The ethnobotanical study of plant species used for medicine by the Egun indigenous tribal group of Lagos State, Nigeria was undertaken with a view to assess the valuable plant species in the area and their methods of preparation. In the recent time, there have been a lot of ethnobotanical studies conducted among the varying ethnic compositions in Nigeria, but a gross dearth of such studies abounds among the Eegun ethnic composition. Several communities were selected, among which 10 respondents were randomly interviewed with the aid of a semi-structured questionnaire guide. Even more, in the LGA, a major market (Badagry market), was chosen where 5 botanical vendors were interviewed on the plant parts sold. A semi-structured questionnaire matrix was used to interview the respondents. All the interviewed were focused, conversational and two-way in communication. Group interviews were conducted in each community to established group consensus on the individual responses provided. The results revealed that a total of 44 plant species, belonging to 38 families, were observed to be valued for medicine and health maintenance. The respondents' indigenous knowledge on these species revealed that diverse diseases were managed with the identified plant species. The parts of the plants used varied, as well as the methods of preparations which were simple, as well as the mode of utilization. Results obtained from the test on the abundance of the identified plant species used in the present study revealed that $7 \%$ of the identified species were very abundant, $41 \%$ were abundant, $41 \%$ were frequent, while $2 \%$ of them were rare. Adequate protection of medicinal plant resources through conservation in their natural reserves is recommended.
\end{abstract}

Keywords: ethnobotanical; medicine; plant species

\section{Introduction}

Ethnobotanical studies today are recognized as the most viable method of identifying new medicinal plant or refocusing on those reported for bioactive constituents (Ogol et al., 2002). The use of plants for treating diseases is as old as human kind. Hence, this has significantly supported primary health care. All cultures from ancient times to the present day have used plants as a source of medicines. Today, as many as $80 \%$ of the world people depend on traditional medicine for their primary health care needs (WHO, 2005). This great surge of public interest in the use of plant as medicines has been based on the assumption that the plant will be available on a continual basis; unfortunately, no concerted efforts are being made to ensure this particularly in the face of the threats posed by increasing demand, a vastly increasing human population and extensive destruction of plant-rich habitats such as the tropical forests, wetlands, Mediterranean ecosystems and parts of the arid zone.

The Eegun tribe is found in Badagry Local Government, Lagos State of Nigeria. The tribe is rich in medicinal lore because of the mangrove nature of the vegetation in the area. Its high humid condition promotes rapid growth of dense vegetation in the area. The use of plants for medicinal purpose is common and widespread among the Eegun people in Badagry (Makinde et al., 2015). Knowledge of traditional medicine is being passed by oral traditions from one generation to another among the indigenes.

In the recent time, there have been a lot of ethnobotanical studies conducted among the varying ethnic compositions in Nigeria, but a gross dearth of such studies abounds among the Eegun ethnic composition. Thus, the present study aimed at assessing the plant species that are valued for use as medicine by the Egun people at Badagry creek, Lagos State, Nigeria. 


\section{Materials and Methods}

Thestudy area

Badagry, the study area, is a coastal town in Badagry Local Government Area (LGA) in Lagos State, Nigeria. It is located between the city of Lagos and Seme, the border town of Benin Republic. According to the 2006 census, the municipality had a total population of 237,731 and covered $442,993 \mathrm{~km}^{2}$ area. The Badagry creek is located on latitude $2.42^{\prime}$ and $3.2^{\prime} \mathrm{E}$ and between longitude 6.23 and $6.28^{\prime} \mathrm{N}$, and forms part of the continuous lagoon that stretches from port Novo to Lagos.

The climate is dominated by heavy rain season which last from April to October. The soil in Badagry is lightly grey sandy type with vegetation over the low lying plains and marshes near the lagoons and creeks. The vegetation is made up of woody plant, shrubs and oil palm trees in the sandy areas, while the marshy areas are covered by mangrove. The mean monthly temperature fluctuates around $30^{\circ} \mathrm{C}$. The relative humidity is high throughout the year and may not be less than 70-80\% around Lagos and other lagoons and seaside locations (Abegunde, 2002) (Figs. $1-2)$.
Methodology

In Badagry Local government area, five rural communities were selected randomly for the study. These communities were Aivoji, Ajido, Gbaji, Sakpo and Sito. In each community, 10 respondents were randomly selected and interviewed with the aid of a semi-structured questionnaire guide. Also, in the LGA, a major market (Badagry market) where 5 botanical vendors were interviewed on the plant parts sold in the market.

A semi-structured questionnaire matrix was used to interview the respondents, which were focused, conversational and two- way in communication. Plant species whose parts where valued for folk medicine were identified. The diseases they cure and /or prevent were identified, as well as plants mode of administration. Similarly, medicinal plant vendors, group of respondents and key informants where identified and interviewed as stated by Adedeji et al. (2018).

The index of wealth used to classify the economic status of respondents was based on the cumulative economic returns from the assets and production of the respondents. Hence, respondents were classified into resource-poor and resource-rich.

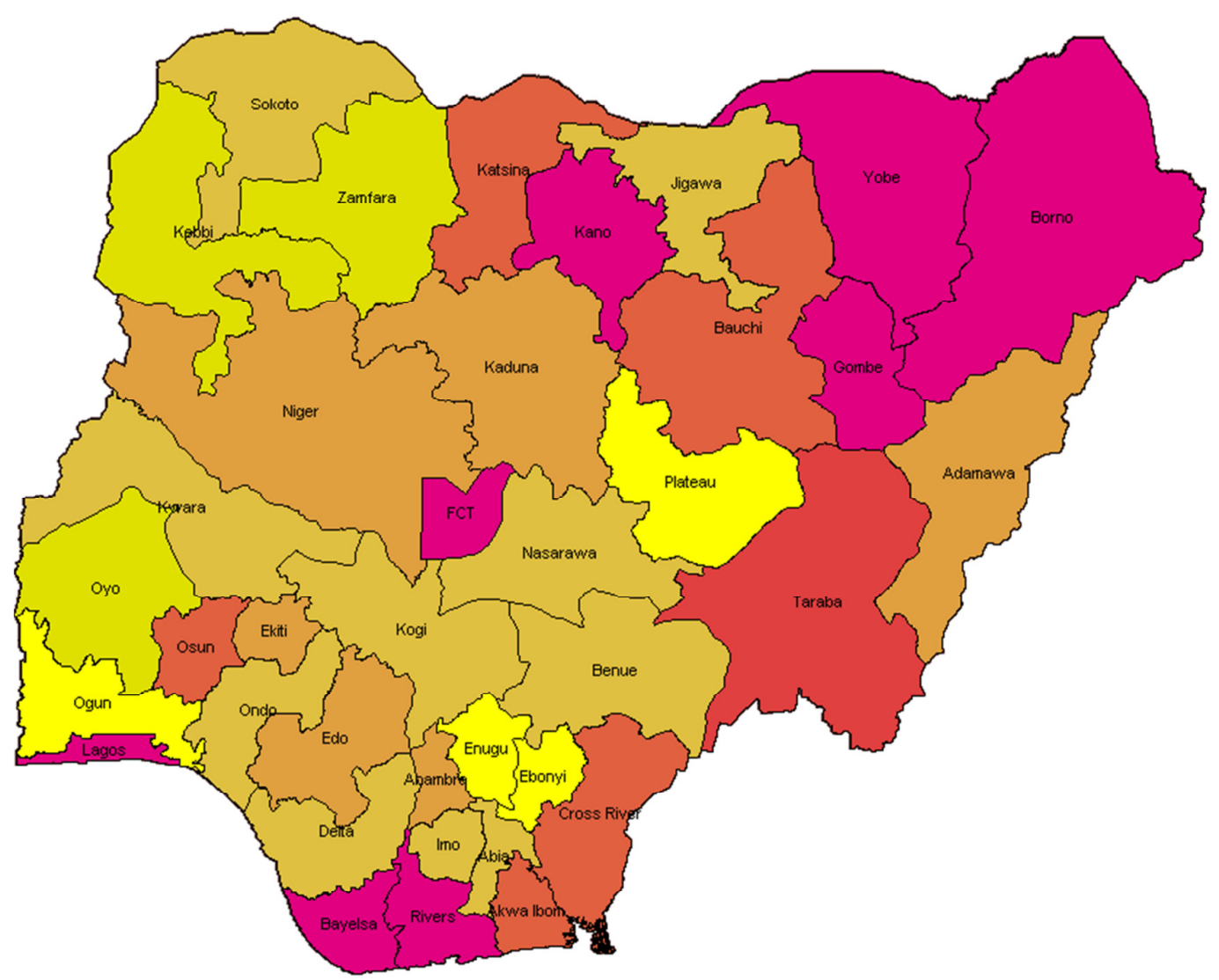

Fig. 1. Map of Nigeria showing Lagos State, Nigeria 
320

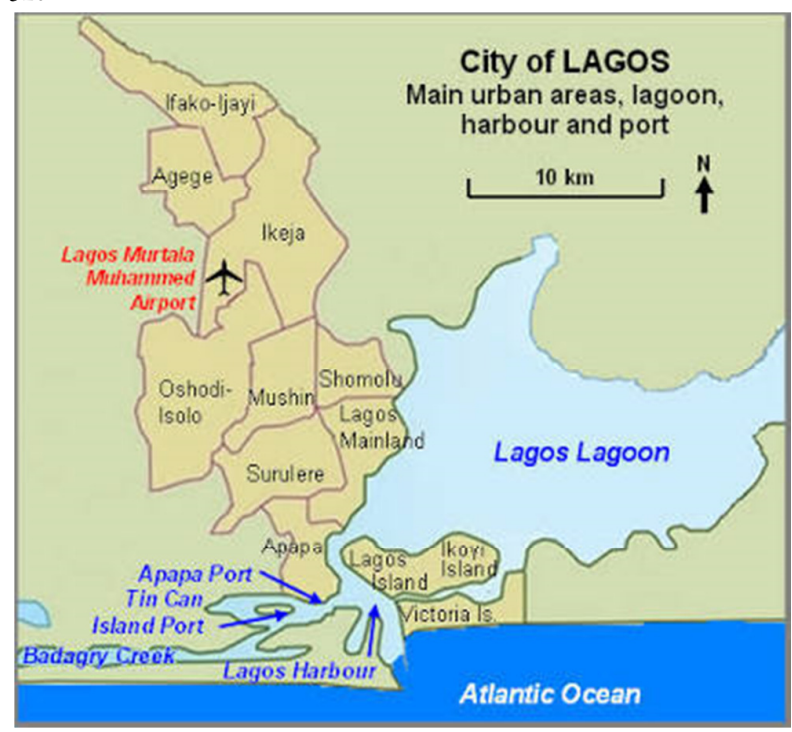

Fig. 2. Map of Lagos State showing Badagry Creek

Group interviews were conducted in each community to established group consensus on the individual responses provided. Key informants consisting of health, forestry and community development officers were identified in each community and interviewed. The botanicals were collected, identified and voucher specimens were kept in the Departments' herbarium.

\section{Results}

Table 1 revealed that the herbal vendors, their clients and resident's respondents transcend sex, age and literacy status, though most of them were females (60\% among vendors, $70 \%$ among clients), adults of ages between 20-50 years ( $60 \%$ of both vendors and clients, respectively) and illiterates (35\%). Field observation revealed that the Eegun people have knowledge of the medicinal values of plants around their environment. The indigenous ethno-botanical knowledge was not documented and the act of transmitting such data from one generation to another is declining.
Trading in medicinal plants is now prominent in the study area and was dominated by females who sourced their stocks from diverse areas within and outside the study area. Field observation further revealed that medicinal plants vendors in the study area could be classified as wholesale and retail vendors. Indigenous knowledge on the composition and prescriptions of the plant derived medicine are now learnt as profession. Thus vendors are now practitioners that attend to numerous patents on daily basis. Various plant parts constituted the ingredients for the plant derived medicine.

A total of 44 plant species, belonging to 38 families, were observed to be valued for medicine and health maintenance (Table 2). While 5 of these species where members of the family Rutaceae, 4 were Annonaceae, 3 were members of the families Anacardiaceae, Asteraceae, Poacea and Rubiaceae. The families Amaranthaceae, Apocynaceae, Asteraceae, Caesapiniaceae, Clusiaceae, Combretaceae, Cucurbitaceae, Euphorbiaceae, Meliaceae and Piperaceae have 2 members each, while other families possess one member each.

Secondary information used in the study revealed the phytochemical constituents present in each of the identified plant species (Table 3). The respondents' indigenous knowledge on these species revealed that diverse diseases were managed with the identified plant species (Table 4).

The parts of the plants used varied. The whole plants of $43 \%$ of the identified species were valued for use as medicine (Table 4), 16\% of the leaves only, $14 \%$ of roots only, $11 \%$ of combined leaves and stems, $7 \%$ of combined roots and stems and 2\% each of flowers only, seeds only, stems only and combines flowers, seeds and leaves were equally valued for medicine.

The methods of preparations were simple and easy to accomplish. The responses (Table 5) revealed that $48 \%$ of the plant-based medicines were prepared by boiling the identified plant parts in water, $30 \%$ by infusion, $18 \%$ by combined infusion and decoction methods and $5 \%$ by blending. The mode of utilization of the plant-derived medicines varied also, it was observed that $84 \%$ of the plant based medicine was utilized by oral administration only, $9 \%$ by bathing only and $7 \%$ by both bathing and oral administration.

Table 1. Socio-economic classification of respondents sampled in Badagry Local Government area of Lagos State

\begin{tabular}{|c|c|c|c|c|}
\hline \multirow{2}{*}{ Feature } & \multirow{2}{*}{ Description } & \multicolumn{3}{|c|}{ Proportion (\%) of respondents } \\
\hline & & Herbal vendor & Clients and Resident & Average, Total \\
\hline \multirow{3}{*}{ Sex } & Male & 20 & 30 & 25 \\
\hline & Female & 80 & 70 & 75 \\
\hline & $<20$ yrs & 0 & 10 & 5 \\
\hline \multirow[t]{2}{*}{ Age } & $20-50 \mathrm{yrs}$ & 60 & 60 & 60 \\
\hline & $>50 \mathrm{yrs}$ & 40 & 30 & 35 \\
\hline \multirow{2}{*}{ Literacy } & Literate & 60 & 70 & 65 \\
\hline & Illiterate & 40 & 30 & 35 \\
\hline \multirow{2}{*}{ Economic status } & Resource Rich & 40 & 50 & 45 \\
\hline & Resource Poor & 60 & 50 & 55 \\
\hline \multirow{2}{*}{ Occupation } & Agricultural & 70 & 80 & 75 \\
\hline & Non - Agricultural & 30 & 20 & 25 \\
\hline
\end{tabular}


Table 2. Identified plant species valued for medicine in Badagry Local Government area of Lagos State

\begin{tabular}{|c|c|c|c|}
\hline $\mathrm{S} / \mathrm{N}$ & Scientific name & Local name & Family \\
\hline 1 & Allophylus africanus & Alloviatan & Sapindaceae \\
\hline 2 & Amaranthus cruentus & Dido beko & Amaranthaceae \\
\hline 3 & Amaranthusspinosus & Oma & Amaranthaceae \\
\hline 4 & Annona muricata & Sharp sharp & Annonaceae \\
\hline 5 & Annona senegalensis & Azun & Annonaceae \\
\hline 6 & Anthrocleista djalonesis & Gusuetin & Logariaceae \\
\hline 7 & Argemone mexicana & Dediyo & Papaveraceae \\
\hline 8 & Calendula officianalis & Aduko & Asteraceae \\
\hline 9 & Calotropis procera(wild) & Awirikomi & Apocynaceae \\
\hline 10 & Carica papaya & Gbantee & Caricaceae \\
\hline 11 & Chrysophylum alibidum & Azanti & Sapotaceae \\
\hline 12 & Cistrus media & Kretin & Rutaceae \\
\hline 13 & Citrus aurantifolia & Yovosan kere & Rutaceae \\
\hline 14 & Citrus aurantum & Zingbo & Rutaceae \\
\hline 15 & Citrus sinensis & Yovosan & Rutaceae \\
\hline 16 & Cola acuminate & Avitin & Sterculiaceae \\
\hline 17 & Corchnus olitorus & Nehun & Malvaceae \\
\hline 18 & Corpolobia lutea & Aviantin & Polygalaceae \\
\hline 19 & Cretera adansonii & Wotozinzen & Capparaceae \\
\hline 20 & Cymbopogon citrates & Tin maa & Poaceae \\
\hline 21 & Desmodium gangeticum & Wovonekun & Myrtaceae \\
\hline 22 & Dialium guineense & Asisoyetin & Fabaceae \\
\hline 23 & Diospyros monbuttensis & a) Adama; b)Egungun eja & Ebenaceae \\
\hline 24 & Gossypium barbademces & Sekanfuntin & Malvaceae \\
\hline 25 & Helianthus annus & Haiyohaiyo & Asteraceae \\
\hline 26 & Hordeum vulgare & a) Isapo b)Igotu & Poaceae \\
\hline 27 & Irvingia gabonensis & Asiyan & Irvingiaceae \\
\hline 28 & Jatropha curcas & Nigbapotin & Euphorbiaceae \\
\hline 29 & Kigelia africana & Ayanpon & Bignoniaceae \\
\hline 30 & Landolphia dulcis & Kavotoetin & Apocynaceae \\
\hline 31 & Lawsonia inermis & Laritin & Lythraceae \\
\hline 32 & Melia azearach & Keketum & Meliaceae \\
\hline 33 & Ocimum graticimum & Chama dido & Labiatae \\
\hline 34 & Psidium guajava & Kekun kekuntin & Myrtaceae \\
\hline 35 & Rauvolfia vomitori & Lema & Apocynaceae \\
\hline 36 & Rhoicissus tridemtata & a) Aviaba b)Viporonba & Vitaceae \\
\hline 37 & Saccharum officinarum & Tetere gungun & Poaceae \\
\hline 38 & Sena alata & Ahinma & Caesalpinaceae \\
\hline 39 & Strophantus hispidus & Dikuyintin & Apocynaceae \\
\hline 40 & Synsepalum dulcificum & Ayiyere & Sapotaceae \\
\hline 41 & Tephrosea purpurea & Fiyo & Fabaceae \\
\hline 42 & Thaumatococcus Danielle & a.Aba b.Afremo & Maranthaceae \\
\hline 43 & Vernonia amygdalina & Aloma & Asteraceae \\
\hline 44 & Zinagiber officinale & Ata ile & Zingiberaceae \\
\hline
\end{tabular}


Table 3. Phytochemical constituents present in the identified plant species used as medicine

\begin{tabular}{|c|c|c|c|}
\hline $\mathrm{S} / \mathrm{N}$ & $\begin{array}{l}\text { Local } \\
\text { name }\end{array}$ & Scientific name & Phytochemicals \\
\hline 1 & Alloviatan & Allophylus africanus & Anthraquinones, carbohydrates, flavonoids, phenols, saponins, tannins. Oladosu et al., 2013 \\
\hline 2 & Dido beko & Amaranthus cruentus & $\begin{array}{l}\text { Cardenoids, carotenoids, saponins, flavonoids, iridoids, steroids, triterpenes, } \\
\text { polyphenols, tannins. Fernand et al., } 2012\end{array}$ \\
\hline 3 & Oma & Amaranthusspinosus & Carbohydrate, cardiac glycosides, flavonoids, phenol, protein, saponins. Khanal et al., 2014 \\
\hline 4 & Sharp sharp & Annona muricata & Alkaloids, cardiac glycosides, flavonoids, reducing sugar, saponins, tannins, triterpenoids. Usunmobun et al., 2014 \\
\hline 5 & Azun & Annona senegalensis & Alkaloids, cardiac glycosides, glycoside, flavonoid, saponins, steroid, tannins, volatile oil. Idayat et al., 2014. \\
\hline 6 & Gusuetin & Anthocleista djalonensis & Alkaloids, flavonoids, saponins, inulins, reducing sugar, tannins, phlobatannins, steroids. Luter et al., 2012 \\
\hline 7 & Dediyo & Argemone mexicana & $\begin{array}{l}\text { Alkaloids, amino acids, anthraquinones, cardiac glycosides, fatty acids, flavonoids, phenols, steroids, tannins, terpenoids. Mergu } \\
\qquad \text { et al., 2017, Apurba et al., } 2012 .\end{array}$ \\
\hline 8 & Aduko & Calendula officianalis & Amino acids, carbohydrates, fatty acids, flavonoids, saponins, sterols, tannins, triterpenoids, phenols. Chakraborthy, 2010 \\
\hline 9 & Awirikomi & Calotropis procera & Alkaloids, cardiac glycoside, flavonoid, saponin, tannins, phenols. Akindele et al., 2017 \\
\hline 10 & Gbantee & Carica papaya & Alkaloids, flavonoids, glycoside, quinones, tannins. Ikeyi et al., 2013 \\
\hline 11 & Azanti & Chrysophylum alibidum & Alkaloids, cardiac glycoside, flavonoid, saponin, tannins, phenols. Okoli et al., 2010 \\
\hline 12 & Kretin & Citrus medica & Alkaloids, carbohydrates, flavonoids, glycosides, phenols, steroids. Kalpesh et al., 2012 \\
\hline 13 & $\begin{array}{c}\text { Yovosan } \\
\text { kere }\end{array}$ & Citrus aurantifolia & Anthraquinones, phenolic compounds, saponins. Emad, 2016 \\
\hline 14 & Zingbo & Citrus aurantium & Alkaloids, flavonoids, glycoside, phenol, saponins, steroid, tannins, terpenoids. Khudhair et al., 2017 \\
\hline 15 & Yovosan & Citrus sinensis & Alkaloids, cardiac glycosides, saponins, tannins, terpenoids. Mamta et al., 2013 \\
\hline 16 & Avitin & Cola acuminate & Alkaloids, anthraquinones, cardiac glycoside, saponins, steroids, tannins, volatile oils. Kanoma et al., 2014 \\
\hline 17 & Nehun & Corchorus olitorus & Alkaloids, flavonoids, saponins, tannins. Sanjida et al., 2015 \\
\hline 18 & Aviantin & Carpolobia lutea & Anthraquinones, cardiac glycosides, flavonoids, saponins, simple sugar, terpenes. Ette et al., 2014 \\
\hline 19 & Wotozinzen & Crateva adansoni & Alkaloids, carbohydrate, flavonoids, saponins, steroids, tannins, terpenoids \\
\hline 20 & Tin maa & Cymbopogon citrates & Carbohydrates, flavonoids, phenols, tannins, volatile oil. Ewansiha et al., 2012 \\
\hline 21 & $\begin{array}{l}\text { Wovoneku } \\
\text { n, Zedari }\end{array}$ & Desmodium gangeticum & Alkaloids, carbohydrates, flavonoids, glycosides, phenols, saponins. Niranjan et al., 2008 \\
\hline 22 & Asisoyetin & Dialium guineense & Alkaloids, flavonoids, glycosides, saponins, tannins. Ajiboye et al., 2015 \\
\hline 23 & $\begin{array}{l}\text { a) Adama } \\
\text { b)Egungun } \\
\text { eja }\end{array}$ & Diospyros monbuttensis & $\begin{array}{l}\text { Alkaloids, anthraquinones, anthocyanins, coumarins, polyphenols, } \\
\text { polyterpene, saponins, sterolds, sterols, quinones. Tuo et al., } 2015\end{array}$ \\
\hline 24 & Sekanfuntin & Gossypium barbademces & Alkaloids, cyanogenic glycosides, flavonoids, saponins, phenolics. Muhammad et al., 2014 \\
\hline 25 & Haiyohaiyo & Helianthus annus & Alkaloids, carbohydrates, flavonoids, steroids, triterpenoids. Rubab et al., 2016 \\
\hline 26 & $\begin{array}{l}\text { a) Isapo } \\
\text { b)Igotu }\end{array}$ & Hordeum vulgare & Alkaloids, flavonoids, fat, glycoside, saponins, phytosterol, protein, reducing sugar, starch, tannins. Kumara et al., 2015 \\
\hline 27 & Asiyan & Irvingia gabonensis & $\begin{array}{l}\text { Cardiac glycosides, carbohydrates, flavonoids, saponins, steroids, } \\
\text { tannins. Efosa et al., } 2016\end{array}$ \\
\hline 28 & Nigbapotin & Jatropha curcas & $\begin{array}{l}\text { Alkaloids, carbohydrates, flavonoids, glycoside, flavonoid, protein, } \\
\text { saponins, sterols, tannins, triterpenoids. Ahirrao et al., } 2011\end{array}$ \\
\hline 29 & Ayanpon & Kigelia Africana & Alkaloids, flavonoids, glycoside, phenolic compound, reducing sugar, tannins. Abdulkadir et al., 2015 \\
\hline 30 & Kavotoetin & Landolphia dulcis & $\begin{array}{c}\text { Alkaloids, flavonoids, glycosides, phenols, saponins, steroids, } \\
\text { tannis. Ramesa et al., } 2016\end{array}$ \\
\hline 31 & Laritin & Lawsonia inermis & Alkaloids, glycosides, saponins, tannins, quinones. Khaled et al., 2016 \\
\hline 32 & Keketum & Melia azedarach & Alkaloids, anthraquinones cyanogenic glycoside, flavonoids, phenols, saponins. Muhammad et al., 2017 \\
\hline 33 & $\begin{array}{l}\text { Chama } \\
\text { dido }\end{array}$ & Ocimum graticimum & Alkaloids, flavonoids, glycosides, saponins, steroids, tannins, terpenoids, phylobatannins. Priscilla, 2016 \\
\hline 34 & $\begin{array}{c}\text { Kekun } \\
\text { kekuntin }\end{array}$ & Psidium guajava & Alkaloids, carbohydrates, flavonoids, saponins, sterols, tannins. Vikrant et al., 2012 \\
\hline 35 & Lema & Rauvolfia vomitoria & Alkaloids, cardiac glycoside, flavonoids, saponin, steroid, terpenoid. Olajumoke et al., 2012 \\
\hline 36 & $\begin{array}{l}\text { a)Aviaba } \\
\text { b)Viporonb } \\
\text { a }\end{array}$ & Rhoicissus tridentate & Alkaloids, flavonoids, phenols, saponins, tannins, terpenoid. Mwangi et al., 2015 \\
\hline 37 & $\begin{array}{l}\text { Tetere } \\
\text { gungun }\end{array}$ & Saccharum officinarum & Alkaloids, hydrogen cyanide, flavonoids, saponins, tannins. Human et al., 2016 \\
\hline 38 & Ahinma & Sena alata & Alkaloids, anthraquinones, carbohydrates, cardiac glycosides, phylobatannins, protein, saponins. Karthika et al., 2016 \\
\hline 39 & Dikuyintin & Strophantus hispidus & Anthraquinone, cardiac glycosides, flavonoids, tannins, terpenoids. Ayoola et al., 2008 \\
\hline 40 & Ayiyere & Synsepalum dulcificum & Alkaloids, flavonoids, cardiac glycosides, polyphenols, tannins, saponin. Osabar et al., 2016 \\
\hline 41 & Fiyo & Tephrosea purpurea & Alkaloids, cardiac glycosides, flavonoids, resins, steroids, tannins, phenols, quinone. Suriyavathana et al., 2014 \\
\hline 42 & $\begin{array}{c}\text { a.Aba } \\
\text { b.Afremo }\end{array}$ & Thaumatococcus Danielle & Alkaloids, cardiac glycosides, flavonoids, phylobatannins, saponins, tannins, terpenoids. Shalom et al ., 2014 \\
\hline 43 & Aloma & Vernonia amygdalina & Alkaloids, flavonoids, glycosides, phenols, reducing sugar, saponins, terpenoids. Ifeanyi et al., 2016 \\
\hline 44 & Ata ile & Zingiber officinale & Alkaloids, cardiac glycoside, flavonoids, reducing sugars, saponins, polyphenols. Osabor et al., 2015 \\
\hline
\end{tabular}


Table 4. Parts used for medicine in the identified plant species in Badagry Local Government, Lagos State

\begin{tabular}{|c|c|}
\hline Parts Used & Identified species \\
\hline Flowers & C. officinalis. Proportion: $2 \%$ \\
\hline Seeds & I.gaboneensis. Proportion: $2 \%$ \\
\hline Leaves & $\begin{array}{l}\text { H. vulgare, T. danielle, C. citratus, C. adansoni, S. dulcificum, A. africanus, C. olitorus. } \\
\text { Proportion: } 16 \%\end{array}$ \\
\hline Stems & S. officinarum. Proportion: $2 \%$ \\
\hline Roots & $\begin{array}{l}\text { Z. officinale, D. guineense, R. vomitoria, L. inermis, O. graticimum, V. amygdalina } \\
\text { Proportion: } 14 \%\end{array}$ \\
\hline Roots and Stems & R. tridentate, C. procera, C. albidum. Proportion: $7 \%$ \\
\hline Flowers, Seeds \& Leaves & H. annus. Proportion: $2 \%$ \\
\hline Leaves \& Roots & T. purpurea, C. papaya, A. muricata, S. hispidus, A. spinosus. Proportion: $11 \%$ \\
\hline Whole plant & $\begin{array}{l}\text { A.segalensis, P. guajava, D. monbuttensis, A. cruentus, } S . \text { alata, M. azedarach, J. curcas, } \\
\text { K. africana, C. medica, C. lutea, C. acuminate, L. dulcis, G. barbademces, A. } \\
\text { Mexicana, D. gangeticum, C. sinensis, C. aurantifolia. } \\
\text { Proportion: } 43 \%\end{array}$ \\
\hline
\end{tabular}

Table 5. Diseases cured, methods of preparations and utilizations of plant medicines obtainable from the identified plant species in Badagry Local Government, Lagos State

\begin{tabular}{|c|c|c|c|c|}
\hline $\mathrm{S} / \mathrm{N}$ & Species & Diseases cured & Methods of preparation & Administration/utilization \\
\hline 1 & Helianthus annus & $\begin{array}{l}\text { Kidney infections, skin infections, } \\
\text { high fevers }\end{array}$ & $\begin{array}{l}\text { Infusion of the leaves; the seeds are crushed to } \\
\text { get the oil }\end{array}$ & $\begin{array}{l}\text { Oral administration, the extract oil of the seeds } \\
\text { are applied on skin surface }\end{array}$ \\
\hline 2 & Rhoicissus tridentate & $\begin{array}{l}\text { a) Prevent abortion } \\
\text { b) Cures fibroid }\end{array}$ & $\begin{array}{l}\text { Decoction and infusion of the root and stem } \\
\text { bark }\end{array}$ & Oral administration \\
\hline 3 & Annona senegalensis & Convulsion, epilepsy & Barks decoction & Oral administration \\
\hline 4 & Hordeum vulgare & $\begin{array}{l}\text { Weakness, lack of shape or vigor in } \\
\text { children }\end{array}$ & Boil in water & The water extract is used to bathe the children \\
\hline 5 & Psidium guajava & Obesity & Decoction in water & Oral administration \\
\hline 6 & Diospyros monbuttensis & Chicken pox, fever, feeling pain & $\begin{array}{l}\text { Decoction or infusion } \\
\text { Boil in water }\end{array}$ & Extract is used to bathe \\
\hline 7 & Calotropisprocera & Fever & Stem and root are rinsed and infused & Chewed orally \\
\hline 8 & Tephrosea purpurea & Weakness, stomach problem & $\begin{array}{l}\text { Decoction of leaves in water; infusion of the } \\
\text { root }\end{array}$ & The extract is used to bathe for children \\
\hline 9 & Caricapapaya & Malarial, typhoid fever & Decoction in water & Oral administration \\
\hline 10 & Saccharum officinarum & Urinary tract infection & Cut the stem pulp and infused in water & The stem pulp is being chewed \\
\hline 11 & Zingiber officinale & $\begin{array}{c}\text { Migraine, gastro intestinal tract } \\
\text { disease, high blood pressure, weight } \\
\text { loss }\end{array}$ & Infusion of the root & The extract of the root is taken orally \\
\hline 12 & Thaumatococcus Danielle & Malarial, fever & Root can be blended & Oral administration \\
\hline 13 & Chrysophylum alibidum & $\begin{array}{l}\text { Yellow fever, malaria skin infection, } \\
\text { gonorrhea, urinary tract infections }\end{array}$ & Root and stem decoction & Oral administration of the bark extract \\
\hline 14 & Amarantus cruentus & Prevention of abortion & Decoction of various shape of the leaves & The extract of the leaves is taken orally \\
\hline 15 & Irvingia gabonensis & Diabetes, heart disease, anemia & Cooking of seeds and eating the fruit raw & Oral administration \\
\hline 16 & Sena alata & Fever, stomach ulcer and pain & Decoction in water & Oral administration \\
\hline 17 & Annona muricata & Rheumatism, diabetes, eczema, boils & $\begin{array}{l}\text { Infusion of the bark in alcohol; decoction of } \\
\text { the leaves }\end{array}$ & Oral administration \\
\hline 18 & Calendula officianalis & Children's fever, skin infection & The flowers are dried, blend into powder & $\begin{array}{l}\text { Apply unto the surface infected or bath with } \\
\text { the water extract }\end{array}$ \\
\hline 19 & Cymbopogon citrates & $\begin{array}{l}\text { Malaria, high blood pressure, } \\
\text { diarrhea, fever }\end{array}$ & Decoction of the leaves & $\begin{array}{l}\text { Oral administration; the extract is used to } \\
\text { bathe }\end{array}$ \\
\hline 20 & Synsepalum dulcificum & Asthma, diabetes, weight loss, cancer & Decoction of the leaves & Oral administration \\
\hline 21 & Melia azedarach & $\begin{array}{l}\text { Asthma, weakness, headache, } \\
\text { diarrhea, nervous disorder }\end{array}$ & Decoction of leaves, stem, and root bark & Oral administration \\
\hline 22 & Jatropha curcas & Asthma, malaria fever, eczema & $\begin{array}{c}\text { Decoction of the root or infusion of the } \\
\text { leaves }\end{array}$ & $\begin{array}{l}\text { The stem is chewed; oral administration of the } \\
\text { plant extract }\end{array}$ \\
\hline 23 & Dialium guineense & $\begin{array}{l}\text { Cough, stomatitis, asthma \& } \\
\text { toothache }\end{array}$ & Infusion & Oral administration \\
\hline 24 & Rauvolfia vomitoria & $\begin{array}{l}\text { Asthma, diarrhea, rheumatism, } \\
\text { jaundice }\end{array}$ & Infusion of the bark & Oral administration \\
\hline 25 & Kigelia africana & $\begin{array}{l}\text { Obesity, digestive disorder, } \\
\text { constipation, veneral diseases, } \\
\text { rheumatism }\end{array}$ & Decoction & $\begin{array}{l}\text { Oral administration; plant extract is applied } \\
\text { externally to rheumatic joint }\end{array}$ \\
\hline
\end{tabular}




\begin{tabular}{|c|c|c|c|c|}
\hline 26 & Citrus aurantium & Obesity, typhoid fever & Infusion & Oral administration \\
\hline 27 & Cretera adansoni & Stomach troubles, syphilis, obesity & Decoction of the bark In lime juice & $\begin{array}{l}\text { Oral administration of the plant extract; the } \\
\text { powdered bark is applied to the swollen part of } \\
\text { the body }\end{array}$ \\
\hline 28 & Strophantus hispidus & $\begin{array}{l}\text { Conjunctivitis, obesity, fever, skin } \\
\text { disease, leprosy and rheumatic } \\
\text { infections }\end{array}$ & $\begin{array}{c}\text { Root infusion in lime juice; decoction of } \\
\text { leaves and bark }\end{array}$ & Oral administration of the plant extract \\
\hline 29 & Anthrocleista djalonesis & $\begin{array}{l}\text { Intestinal problems, malaria, } \\
\text { jaundice, skin infections, hernia }\end{array}$ & Decoction of the plant parts. & Oral administration \\
\hline 30 & Desmodium gangeticum & $\begin{array}{l}\text { Rheumatism, pain, diabetes, chronic } \\
\text { fever, diarrhea, asthma, dysentery }\end{array}$ & Infusion of the plant parts. & Oral administration \\
\hline 31 & Citrus medica & $\begin{array}{l}\text { Diabetes, scurvy, kidney stone } \\
\text { disease }\end{array}$ & Infusion & $\begin{array}{l}\text { Oral administration of the fruit juice and plant } \\
\text { extract }\end{array}$ \\
\hline 32 & Carpolobia lutea & Male infertility & Infusion of the root and plant part in alcohol & Oral administration \\
\hline 33 & Cola acuminate & $\begin{array}{l}\text { Stomach ulcer, piles, male infertility, } \\
\text { dysentery, diarrhea }\end{array}$ & Infusion of the plant part & $\begin{array}{l}\text { Oral administration of the plant extract; the } \\
\text { nut is chewed raw }\end{array}$ \\
\hline 34 & Allophylus africamus & $\begin{array}{c}\text { Fever, male infertility, diarrhea, pile, } \\
\text { venereal diseases }\end{array}$ & Decoction in water or pap broth & Oral administration \\
\hline 35 & Landolphia dulcis & $\begin{array}{l}\text { Female infertility, arthritis, sore and } \\
\text { kidney pains. }\end{array}$ & Decoction of the plant barks and the leaves & Oral administration \\
\hline 36 & Gossypium barbademces & $\begin{array}{l}\text { Female infertility, typhoid fever, high } \\
\text { blood pressure }\end{array}$ & Decoction of the plant parts & Oral administration \\
\hline 37 & Argemone mexicana & $\begin{array}{l}\text { Skin infections, Female infertility } \\
\text { and leprosy }\end{array}$ & Decoction of the plant barks & Oral administration \\
\hline 38 & Citrus sinensis & $\begin{array}{l}\text { Fever, catarrh, asthma, high blood } \\
\text { pressure and liver ailment }\end{array}$ & Decoction of the plant & Oral administration of the plant extract \\
\hline 39 & Citrus aurantifolia & Diabetes, weight loss, scurvy & Decoction of the plant parts & Oral administration \\
\hline 40 & Amarantus spinosus & Gonorrhea, eczema, haemorroids & $\begin{array}{l}\text { Decoction of the roots and leaves; the plant is } \\
\text { also burnt to ashes. }\end{array}$ & $\begin{array}{l}\text { The plant extract is taken orally, and the plant } \\
\text { ashes is used to wash sores }\end{array}$ \\
\hline 41 & Corchrus olitorus & $\begin{array}{l}\text { Diabetes, aches and pains, dysentery, } \\
\text { fever, piles, gonorrhea }\end{array}$ & $\begin{array}{l}\text { Leaves are cooked as vegetables; cold infusion } \\
\text { of the leaves }\end{array}$ & $\begin{array}{l}\text { Oral administration of the extract and the } \\
\text { leaves are eaten when cooked }\end{array}$ \\
\hline 42 & Lawsonia inermis & $\begin{array}{c}\text { Malaria \&fever, dysentery, diarrhea, } \\
\text { sore throat, liver problems, } \\
\text { toothache }\end{array}$ & Decoction of the bark; infusion of the leaves & Oral administration \\
\hline 43 & Ocimum graticimum & $\begin{array}{l}\text { Fever, diarrhea, colds, impotence, } \\
\text { dysentery and rheumatism }\end{array}$ & $\begin{array}{l}\text { Infusion of plant parts in water, the leaves are } \\
\text { squeezed in water to get the leaf extract }\end{array}$ & Oral administration \\
\hline 44 & Vernonia amygdalina & $\begin{array}{l}\text { Malarial, fever, diarrhea, dysentery, } \\
\text { hepatitis and cough }\end{array}$ & Infusion of the plant parts in water & Oral administration \\
\hline
\end{tabular}

Table 6. Abundance of the identified plant species in Badagry Local Government Area, Lagos State, Nigeria

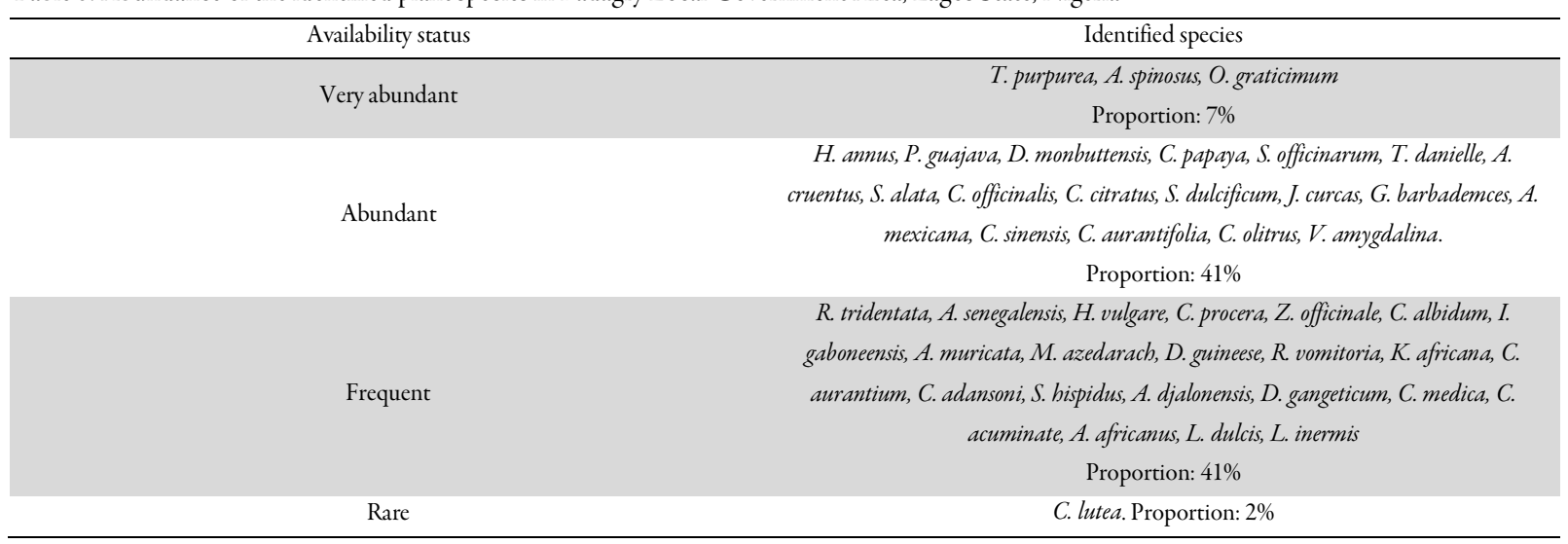

Table 7. Habits of the identified plant species used for medicinal use

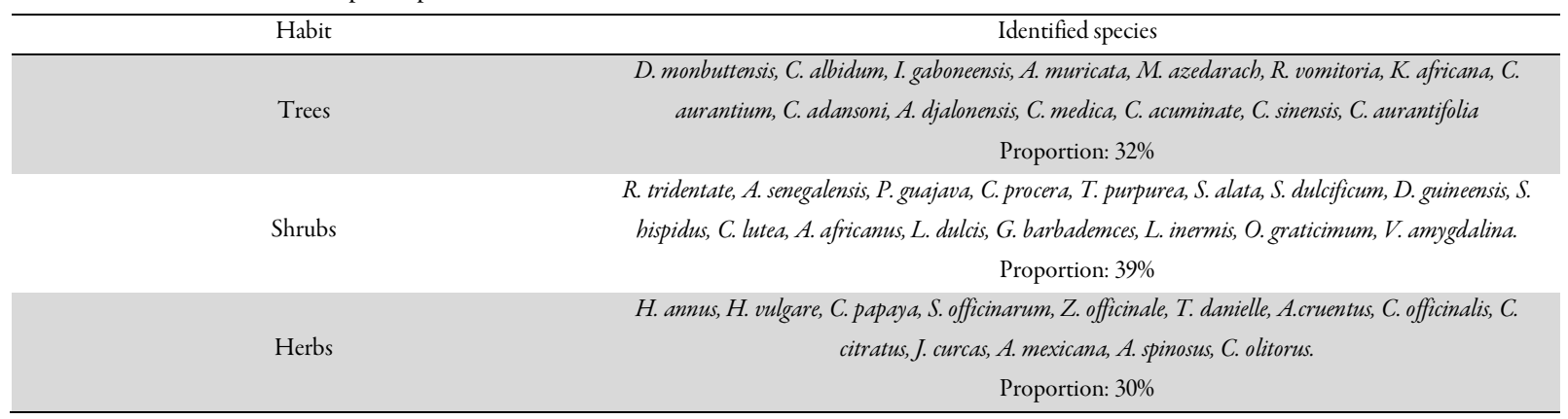


Results obtained from the test on the abundance of the identified plant species used in the study (Table 6) revealed that $7 \%$ of the identified species were very abundant, $41 \%$ were abundant, and $41 \%$ were frequent, while $2 \%$ of them were rare. Table 7 shows the habit of the plant species: $32 \%$, $39 \%$ and $30 \%$ of the identified species were trees, shrubs and herbs respectively.

\section{Discussion}

The study revealed that Eegun, like other tribes in Nigeria (Kayode et al., 2017), still valued the use of plants for health maintenance and management. Medicinal plants are increasingly recognized worldwide as an alternative source of efficacious and inexpensive medications to synthetic chemo-therapeutic compound (Omogbadegun $e t$ al., 2011). Field observation shows that indigenous knowledge on these plants is been passed to the younger generation (Table 1) and the varying socio-economic classification of the respondents does not serve as prerequisites to this transmission. The trading and apprenticeship in medicinal plants observed in the present study tends to suggest that dependence on the use of medicinal plants will be sustained in the study area. Previous study by Ekanem and Udoh (2009) asserted that plants now constitute a major economic resource of most countries of the world including Nigeria.

The intimate consciousness demonstrated by respondents on the medicinal values of the identified species in the study area is further buttressed by the results obtained from the secondary sources that the identified species were rich in phytochemicals (Tables 2 and 3). Ayoola et al. (2008) asserted that these phytochemicals have beneficial effects on health and play active roles in amelioration of diseases. The diversity in the parts of the identified plant valued for medicine tends to lend credence to the assertion of Osabor et al. (2016) that varying quantities of the phytochemicals abounds in the plant parts. Similarly, diversity abounds in such plants species, diseases managed by the plants and the methods of administration of the plant medicine in this study. Field observation revealed that quite often, only the medicinal ingredients (the plant parts) are obtained from the vendors in the markets, while the client prepares the medicine. The methods of preparations were simple without any technology involved. Kayode and Odesola (2017) made similar observation and asserted that this constitutes positive incentive to the preference for plant medicine and the cheap cost of the plant medicine. Also, Makinde et al. (2015) observed that many herbal vendors, trado-medical practitioners have Western education with background in diverse disciplines. Many undergo tutelage as apprentice for several months and some attended conferences and workshops. All these have resulted in better preparations, packaging and thus the efficacies of the plant medicines in the recent times.

Most of the identified plant species were presently 'frequent' on the abundance scale used in the current study (Table 7) with $2 \%$ of the species already attained the 'rare' status. Most of the species were shrubs and trees. Thus, with the parts of the plants used including whole plants, roots, stems, barks, leaves, flowers, fruits and seeds, as well as the increase awareness and preference for plant derived medicine coupled with rapidly developing industrialization and conversion of vegetation to housing and other economic activities in the study area, the need for conservation (Kayode et al., 2015) of most of the identified species cannot be over-emphasized.

\section{Conclusions}

Conservation ensures the availability and sustainability of the identified species for the present and future generations of the inhabitants of the study area. It is therefore necessary to preserve the plant diversity of the vegetation of the study area. Some of the wild medicinal plants should be domesticated. Similarly, sustainable harvesting techniques should be practiced. Part of the existing vegetation in the study area should be constituted as botanical gardens so that adequate protection of medicinal plant resources through conventional conservation in their natural reserves could be attained.

\section{References}

Abegunde MA (2002). Aspect of the physical environment of Lagos. History and People of Lagos state. Lantern Publications Limited.

Abdulkadir MN, Adedokun A,EfosaJ (2015). Phytochemical composition and antimicrobial evaluation of Kigelia Africana LAM. Asian Journal of Plant Science and Research 5(1):1417.

Adedeji DE, KayodeJ, Oyedeji A, Olanipekun MK (2018). Bark extravitism and health maintenance among ljaw Tribal community of Bayelsa State, Nigeria. SaudiJournal ofLife Sciences3(2):111-120.

Ahirrao RA, Patel MR, Pokal DM, Patil JK, Suryawanshi HP (2011). Phytochemical screening of leaves of Jatropha curcas plant. International Journal of Research in Ayurveda and Pharmacy 2(4):13241327.

Ajiboye AE, Ameen MT, Adebayo MR (2015). Antimicrobial activity and phytochemical screening of the fruit pulp of Dialum guineense (Velvet Tamarind) on some microbial isolates. Journal of Microbiology and Antimicrobials 7(4):33-41.

Akindele PO, Fatunla OA, Ibrahim KA, Afolayan CO (2017). Antibacterial and phytochemical screening of Calotropis procera leat extracts against vancomycin and methicillin resistant bacteria isolated from wound samples in hospital patients. Journal of Complementary and alternative Medical Research 2(1):1-14.

Apurba SA, Ahmed HA, Ferdous A, Bhuyan SH, Matin M, Hossain MF (2012). Phytochemical analysis and bioactivities of Argemone mexicana Linn Leaves. Pharmacology online 3:16-23.

Ayoola GA, Folawewo AD, Adesegun SA, Abioro OO, Adepoju BA, Coker HA (2008). Phytochemical and antioxidant screening of some plants of Apocynaceae from south west Nigeria. African Journal of Plant Science 2(10):124-128.

Behera DK, Nath NA (2005). Cross-pollination and critique; Resource conservation and utilization through indigenous knowledge in a tribal community of Orissa-India Indilinga African Journal of Indigenous Knowledge Systems 4:210-268.

Chakraborthy GS (2010). Phytochemical screening of Calendula officinalis LINN leaf extract by TLC. International Journal of Research 1(1):131- 
326 134.

Efosa GE, Emanuel U, Usnomena U (2016). Phytochemical composition, in vitro antioxidant activity and acute toxicity of Irvingia gabonensis bail ethanolic leaf extract. International Journal of Biological Research 4(1):36-41.

Ekanem AP, Udoh FV (2009). The diversity of medicinal plants in Nigeria: An overview. African Natural Products: New Discoveries and Challenges in Chemistry and Quality, ACS Symposium series. American Chemical Society 1021:135-137.

Emad MA (2016). Preliminary phytochemical and antibacterial screening of methanolic leaf extract of Citrus aurantifolia. Pharmaceutical BiotechnologyCurrent Research 1(10):2-5.

Ette E, Nwafor P (2014). In vitro anti- microbial activities of extracts of Carpolobia lutea root. Pakistan Journal of Pharmaceutical Sciences 22(3):335-338.

Ewansiha JU, Garba SA, Mawak JD, Oyewole OA (2012). Antimicrobial activity of Cymbopogon citratus (lemon grass) and its phytochemical properties. Frontiers in Science 2(6):214220.

Fernand WN, Adama H, Jeanne FM, Odile GN (2014). Phytochemical composition, antioxidant and xanthine oxidase inhibition activities of Amaranthus cruenthus L. and Amaranthus bybridus L. extracts. Pharmaceuticals 5(6):613-628.

Gardzirayi CT, Mutandwa EF, ChihiyaJ, Chikosha M (2008). Indigenous knowledge systems in sustainable utilization of wetlands I communal areas of Zimbabwe, a case of Hwedza District. Journal of Agricultural Research 1(4):131-137.

Hugh C, Maduka C, Akubugwo EI, Olorunnipe O, Aloysius NO, Maduka AA, Uzoma C, Aguoru C, Emmanuel UC (2016). Elemental composition and phytochemical screening of aqueous leaf extract and stem bark Extract of Crateva adansoni. International Journal of Biochemistry Research 10(4):1-7.

Idayat SI, Samira A, Musiliu A (2014). Extraction and phytochemical screening of the root and leaf of Annona senegalensis (wild custard apple). Academic Journal of Interdisciplinary studies 3(7):9-15.

Ifeanyi OO, Chikezie OD, Anyi R, Oshim O, Ezugwu UM, Urama EU (2016). Kinetics of minimum inhibitory concentration. Minimum bactericidal concentration and minimum fungicidal concentration of Vernonia amygdalina in microorganisms isolated from wound infection. International Journal of Surgical Research 5(1):8-14.

Ikeyi AP, Ogbonna AO, Ann O, Eze FU (2013). Phytochemical analysis of pawpaw (Carica papaya) leaves. International Journal of Life Sciences Biotechnology and Pharmaceutical Research 2:2250-3137.

Williams IO, Onyenweaku EO, Atangwho IJ (2016). Nutritional and antimicrobial evaluation of Saccharum officinarum consumed in Calabar, Nigeria. African Journal of Biotechnology 15(33):1789-1795.

Kalpesh P, Krutika J, Nishteswa K (2012). A review on phytochemical and pharmacological properties of Citrus medica Linn. International Journal of pharmaceutical and Biological Archives 3(60:1292-1297.

Kanoma AI, Muhammad IS, Abdullahi KS, Maishnu HM, Isah AD (2014). Qualitative and quantitative phytochemical screening of Cola acuminate. Journal of Biology, Agriculture and Health care 4(5):89-97.

Karthika C, Mohamed RK, Manivannan S (2016). Phytochemical analysis and Evaluation of antimicrobial potential of Senna alata LINN. leaves extract". Asian Journal of Pharmaceutical and Clinical Research 9(2):253-257.

Kayode J, Omotoyinbo MA, Ayeni MJ, Oyedeji AA (2015). Stem barks and root extravitism in Ekiti State, Nigeria: Need for conservation as a sustainable innovation in healthcare management in rural area. American Journal of Bioscience 3(2):28-33.

Kayode, J, Arilewo, K, Ayeni MJ (2016). Potential of myths in Ijesa indigenous community of Nigeria in the conservation of her Forest. Bangdalesh.Journal of Botany 45(5):1151-1156.

KayodeJ, AmooJO, Ayeni MJ (2017). Can the bark extravitismamong the aboriginal Yoruba populace of kwara State, Nigeria be sustained? International Journal of development and Sustainability 6(9):10251035.

Kayode J, Odesola FA (2017). Study on the use of indigenous ecological knowledge in the control of vegetable pests in Ekiti State, Nigeria. International Journal of Agricultural Papers 21:13-18.

Khaled SA, Fuad AA, Khaldon O, Alshakka M (2016). Phytochemical screening and antibacterial activity of Yemeni Henna (Lawsonia inermis) against some bacterial pathogens. Journal of Pharmacy and Biological Sciences 11:2427.

Khanal DP, Rant B, Dangol KS (2014). Phytochemical screening, pharmacognostic evaluation and biological activity of Amaranthus spinosus L.Journal of Medicinal Maintenance 1(4):29-34.

Khudhair AM, Abed AL, Tareq RM, Osama KJ (2017). Phytochemical analysis and inhibitory effects of Citrus aurantium L. (Bitter Orange) leaves on some Bacteria isolates in vitro. Diyala Journal of Pure Sciences 13(1):115-126.

Luter L, Rapheal AO, Galadina A, Okoronkwo MU (2012). Phytochemical screening and anti- microbial activity studies of the root extract of Anthocleista djalonensis (Cabbage Tree). International Journal ofChemistry $4(4): 37$.

MakindeSCO, Ojekale AB, Oshinaike TS, Awusinu TS (2015). An ethnomedicinal and ethnobotanical survey of plants herbal therapy used for obesity, asthma, diabetes and fertility by the Badagry people of Lagos State, Nigeria. Journal of Medicinal Plant Studies 3(5):01-06.

Arora M, Kaur P (2013). Phytochemical screening of orange peel and pulp. International Journal of Research in Engineering and Technology 2:12.

Mergu P, Satya PV (2017). Preliminary phytochemical analysis and oral acute toxicity study of the root of Argemone mexicana LINN. International Journal of Research and Development in Pharmacy and LifeSciences 5(2):2010-1017.

Muhammad KA, Mahmood A, Kashif B, Naila A (2017). Anti-fungal, Antioxidant and phytochemical screening of Melia azedarach flower extracts by using different solvents. Journal of Pharmaceutical Research International 20(1).

Muhammad Z, Masanawa AA, Pyeng AK (2014). Phytochemical and mineral analysis of methanoic extracts of Gossypium barbadense. Annals of Experimental Biology 2(4):11-15.

Muhando JA (2005). Cross- pollination critique, sacred sites and environmental conservation: A case of Kenya. Indilinga. African. Journal of Indigenous Knowledge System 4(1):228-242. 
Mwangi JM, Njagi EN, Piero NM, Njagi JM, Agyirifo SD, Gathumbi KP, MuchungiNA (2010). In vitro anti-diabetic effects of Rhoicissus tridenta in Alloxan induced diabetic mice. Journal of Developing Drugs 4(3):2-5.

Niranjan A, Tewari SK (2008). Phytochemical composition and antioxidant potential of Desmodium gangetichum (Linn). Natural Product Radiance 7(1):35-39.

Odora A, Hoppers CA (2004). Indigenous knowledge and the integration of knowledge systems; New Africa Books: Cllaremont, Kaapstad, South Africa.

Ogol C, Ogola P, Odede W, Khayota B (2005). Indigenous knowledge of medicinal and utilization plants Mfangano island, Lake Victoria, Kenya, East Africa.Journal of Science 4:11-28.

Okoli BJ, Okere OS (2010). Antimicrobial activity of the phytochemical constituents of Chrysophyllum albidum DON HOLL (African star apple) plant. Journal of Research in National Development 8(1):1035-1037.

Oladosu IA, Balogun SO, Ademowo GO (2013). Phytochemical screening, antimalarial and histopathological studies of Allophylus africanus and Tragia benthamii. Chinese Journal of Natural Medicines 11(4):371376.

Olajumoke OO, Ajayi SS, Owolabi LO (2012). Phytochemical screening, anti- nutrient composition, proximate analyses and the antimicrobial activities of the aqueous and organic extracts of Rawvolfia vomitoria and leaves of Peperoma pellucida. International Research Journal of Biochemistryand Bioinformatics 2(6):127-134.

Omogbadegun Z, Uwadia C, Ayo C, Mbarika V, Omoregbe N, Otofia E, Chieze F (2011). Multimedia based medicinal Plants Sustainability Management System. International Journal of Computer Science Issues $8(5): 3-7$.

Osabor VN, Bassey FI, Umoh UU (2016). Phytochemical screening and quantitative evaluation of nutritional values of Zingiber officinale (Ginger). American Chemical Science Journal 8(4):1-6.

Osabor VN, Etiuma RA, Ntinya MU (2016). Chemical profile of leaves and roots of miracle fruit (Synsepalum dulcificum). American Chemical Science Journal 12(1):1-8.

Priscilla A (2016). Phytochemical screening and mineral composition of the leaves of Ocimum gratissimum (scent leaf). International Journal of Applied Sciences and Biotechnology 4(2):161-165.

Bhat RS, Al-Daihan S (2014). Phytochemical constituents and antibacterial activity of some green leafy vegetables. Asian Pacific Journal of Tropical Biomedicine 4(3):189-193.
Rasethe MT, Semenya S, Potgieter MJ, Maroyi A (2014). The utilization and management of plant resources in rural areas of the Limpopo Province, South Africa.Journal ofEthno-medicine 9(1):27.

Richmond L, Middleton BR, Gilmer R(2013). Indigenous studies speak to environmental management. Environment Management 52(5):10411045.

Rubab TI, Hossain MM, Majumder K, Amjad H (2016). In vitro phytochemical investigation of Helianthus annus seeds. Bangdalesh Pharmaceutical Journal 19(1):100-105.

Sanjida P, Moytry M, Sanjana R, Awuj K, Sanjida H, Mohammed R(2015). Phytochemical analysis of some medicinal plants. Journal of Applied Pharmaceutical Science 5(9):68-71.

Shalom NC, Adetayo YO, Popoola ST, Bolaji JD, Epelle TT (2014). Analysis of the leaf, fruit and seed of Thaumatococcus daniellii (Benth): Exploring potential uses. Pakistan Journal of Biological Sciences 17(6):849-854

Suriyavathana M, Suganya M, Priya K (2014). Phytochemical screening of Tephrosia purpurea. International Journal of Pharmaceutical Development and Technology 4(4):267-270.

Tuo K, Beorourou S, Toure AO, Quattara K, Silue KD, Tawo KD, et al., Coulibaly A (2015). Phytochemical screening and polyphenolic contents of Dialium dinklagei \& Diospyros monbuttensis, two Ivorian medicinal plants to treat malaria. Journal of Advances in Medical and Pharmaceutical Sciences 2(4):144153.

Turner NJ, Ignance MB, Ignance RT (2000). Traditional and ecological knowledge and wisdom of aboriginal people in British Columbia. Journal ofEcology Applied 10(1):1275-1287.

Usunobun U, Okolie NP, Anyanwu OG, Adegbegi AJ (2014). Phytochemical screening and proximate composition of Annona muricata leaves. European Journal of Botany Plant Science and Pathology 2(1):18-28.

Vikrant A, Narender T, Kashjap CP (2010). Preliminary phytochemical analysis of the extracts of Psidium guajava leaves. Journal of Phamacognosyand Phytochemistry 1(1):112-117.

WHO (2005). National policy on traditional medicine and regulation of herbal medicines: Report of a WHO Global Survey. World Health Organization, Geneva, Switzerland. 